\title{
The population-based Barcelona-Asymptomatic Intracranial Atherosclerosis Study (ASIA): rationale and design
}

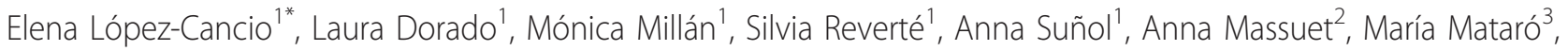
Amparo Galán ${ }^{4}$, Maite Alzamora ${ }^{5}$, Guillem Pera ${ }^{5}$, Pere Torán ${ }^{5}$ Antoni Dávalos', Juan F Arenillas ${ }^{6}$

\begin{abstract}
Background: Large-artery intracranial atherosclerosis may be the most frequent cause of ischemic stroke worldwide. Traditional approaches have attempted to target the disease when it is already symptomatic. However, early detection of intracranial atherosclerosis may allow therapeutic intervention while the disease is still asymptomatic. The prevalence and natural history of asymptomatic intracranial atherosclerosis in Caucasians remain unclear. The aims of the Barcelona-ASymptomatic Intracranial Atherosclerosis (ASIA) study are (1) to determine the prevalence of ASIA in a moderate-high vascular risk population, (2) to study its prognostic impact on the risk of suffering future major ischemic events, and (3) to identify predictors of the development, progression and clinical expression of this condition.

Methods/Design: Cross-over and cohort, population-based study. A randomly selected representative sample of 1,503 subjects with a mild-moderate-high vascular risk (as defined by a REGICOR score $\geq 5 \%$ ) and with neither a history of cerebrovascular nor ischemic heart disease will be studied. At baseline, all individuals will undergo extracranial and transcranial Color-Coded Duplex (TCCD) ultrasound examinations to detect presence and severity of extra and intracranial atherosclerosis. Intracranial stenoses will be assessed by magnetic resonance angiography (MRA). Clinical and demographic variables will be recorded and blood samples will be drawn to investigate clinical, biological and genetic factors associated with the presence of ASIA. A long-term clinical and sonographic followup will be conducted thereafter to identify predictors of disease progression and of incident vascular events.

Discussion: The Barcelona-ASIA is a population-based study aiming to evaluate the prevalence and clinical importance of asymptomatic intracranial large-artery atherosclerosis in Caucasians. The ASIA project may provide a unique scientific resource to better understand the dynamics of intracranial atherosclerosis from its early stages and to identify new potential therapeutic targets for this condition.
\end{abstract}

\section{Background}

Atherosclerosis is a systemic disease with multifactorial etiology now considered the primary cause of morbidity and mortality in developing countries.

Large artery intracranial atherosclerosis disease (ICAD) is a major public health problem as it is probably the major cause of stroke worldwide and consequently, a main cause of long-term disability and mortality [1]. Accumulating evidence suggests that

\footnotetext{
* Correspondence: elenacancio@gmail.com

'Department of Neurosciences, Hospital Universitari Germans Trias I Pujol,

Universitat Autònoma de Barcelona, Badalona, Barcelona, Spain

Full list of author information is available at the end of the article
}

ICAD can also contribute to the development of cognitive impairment and Alzheimer disease although more studies are needed to establish this association [2-5]. Even so, it is a relative neglected frontier [1]. Despite the extended use of non invasive diagnostic techniques as transcranial Doppler (TCD), transcranial color-codedduplex (TCCD), magnetic resonance angiography (MRA) or computed tomography angiography ICAD is an infradiagnosed and understudied disease when compared to extracranial atherosclerosis.

When it turns to symptomatic, intracranial atherosclerosis is a dynamic and aggressive disease. The rate of stroke recurrence is high (up to $18 \%$ in $>70 \%$ stenosis) despite

\section{() Biomed Central}


medical treatment [6-10], and the best therapy for symptomatic intracranial atherosclerosis still remains unknown [11]. In this context, there is a need to increase our knowledge about basic mechanisms and dynamics of intracranial atherosclerosis progression from its preclinical stage.

Intracranial atherosclerosis is not an isolated disease, but related to generalized atherosclerosis affecting other territories as carotid, coronary or peripheral artery disease $[12,13]$. One essential aim in primary prevention is to find tools to improve the evaluation of "the vulnerable patient" $[14,15]$. Classic vascular risk functions (as Framingham) are now being combined with new markers as carotid intima-media thickness, ankle-arm index or CRP values [16] to assess individual vascular risk. Therefore, the identification of new blood, genetic or instrumental biomarkers for asymptomatic atherosclerosis may be crucial to predict and prevent future ischemic events.

Traditional approaches have attempted to target ICAD when it is already symptomatic so there is a lack of studies in the asymptomatic stage. Population studies aimed to determine its prevalence and related vascular risk factors have only been developed in Asian populations, using transcranial Doppler to assess the presence of stenosis [17-20]. Therefore, the prevalence of asymptomatic intracranial atherosclerosis remains unknown in Caucasians.

In this context of uncertainty regarding the prevalence and clinical importance of asymptomatic intracranial atherosclerosis in Caucasians, we designed a prospective study called Barcelona-ASIA (ASymptomatic Intracranial Atherosclerosis), aimed (1) to determine the prevalence of asymptomatic intracranial atherosclerosis in a randomly selected Caucasian population with moderatehigh vascular risk; (2) to study its prognostic impact on the risk of suffering future major ischemic events and/or cognitive decline; and (3) to identify predictors of the development, progression and clinical expression of this condition.

This article describes the protocol details of the Barcelona-ASIA study.

\section{Methods/Design}

This is a population-based, prospective, long-term follow-up observational study that will include over a thousand randomly selected healthy subjects exposed to vascular risk factors and without history of stroke or ischemic heart disease. The study will have two phases, cross-sectional and longitudinal. In the first crosssectional phase, all study subjects will undergo an extensive clinical, laboratory, ultrasound, neuropsychological and neuroimaging protocol in order to determine the prevalence of asymptomatic intracranial atherostenoses among the study population and to identify clinical, biological and genetic associated factors. In the second phase, a long-term clinical and TCCD follow-up will be performed with the purpose of determining the impact of asymptomatic intracranial atherosclerosis on the incidence of major vascular events and cognitive impairment.

\section{Subject selection}

This study will be carried out in the Germans Trias i Pujol University Hospital, a public health tertiary centre of the Barcelonès Nord and Maresme region (Catalonia, Spain), and it is coordinated with the regional Primary Health Care network. The protocol has been approved by the Ethics Committee of our institution.

A rural and urban population of approximately 600.000 residents integrates our metropolitan area. This entire population is registered in a database of the Primary Care Information Technology System (SIAP). A sample of 3010 subjects older than 50 years was randomly selected in 2007 within the PERART study, an ongoing trial that aims to estimate the prevalence and prognosis impact of the peripheral artery disease in our population. Selection of the PERART cohort is described in detail elsewhere [21]. Framingham and REGICOR (Framingham calibrated for Spanish population [22]) functions were previously calculated in all these participants at the initial visit of PERART study.

The ASIA cohort derives from this PERART cohort. From the initial PERART sample of 3010 subjects, we will evaluate the 1503 subjects who met the following inclusion criteria: (1) No history of stroke or transient ischemic attack; (2) No history of coronary disease; (3) Exposure to a light-moderate-high vascular risk, assessed by a REGI$\mathrm{COR} \geq 5$ (which is equivalent to a Framingham $>10$ ); (4) Absence of institutionalization, severe disability or previous chronic neurological/psychiatric disease.

First, on February 2007 a letter was sent to all 1503 selected participants warning them about the study and the chance of being called to take part in it. They were given a 15-day period to express their refusal to collaborate in the study, but no patient denied participation at that time. Subsequently, all possible participants will be contacted by phone (up to five calls) during the recruitment phase. A subset of this initial sample may reject to take part in the study when being informed about the conditions of the study during this phone call. Finally, among the subjects who will accept to come to our hospital for the baseline visit, some will be excluded after reassessment of inclusion criteria (Figure 1). We estimate that about 1000 subjects may complete the study, assuming a $10 \%$ of exclusions and a $20 \%$ of not-accepting participants.

\section{Baseline procedures at initial visit in our centre (Figure 2)} 1. Signing of informed written consent

2. Clinical data collection. Clinical data of every subject will be collected in a questionnaire specifically designed for this study: 


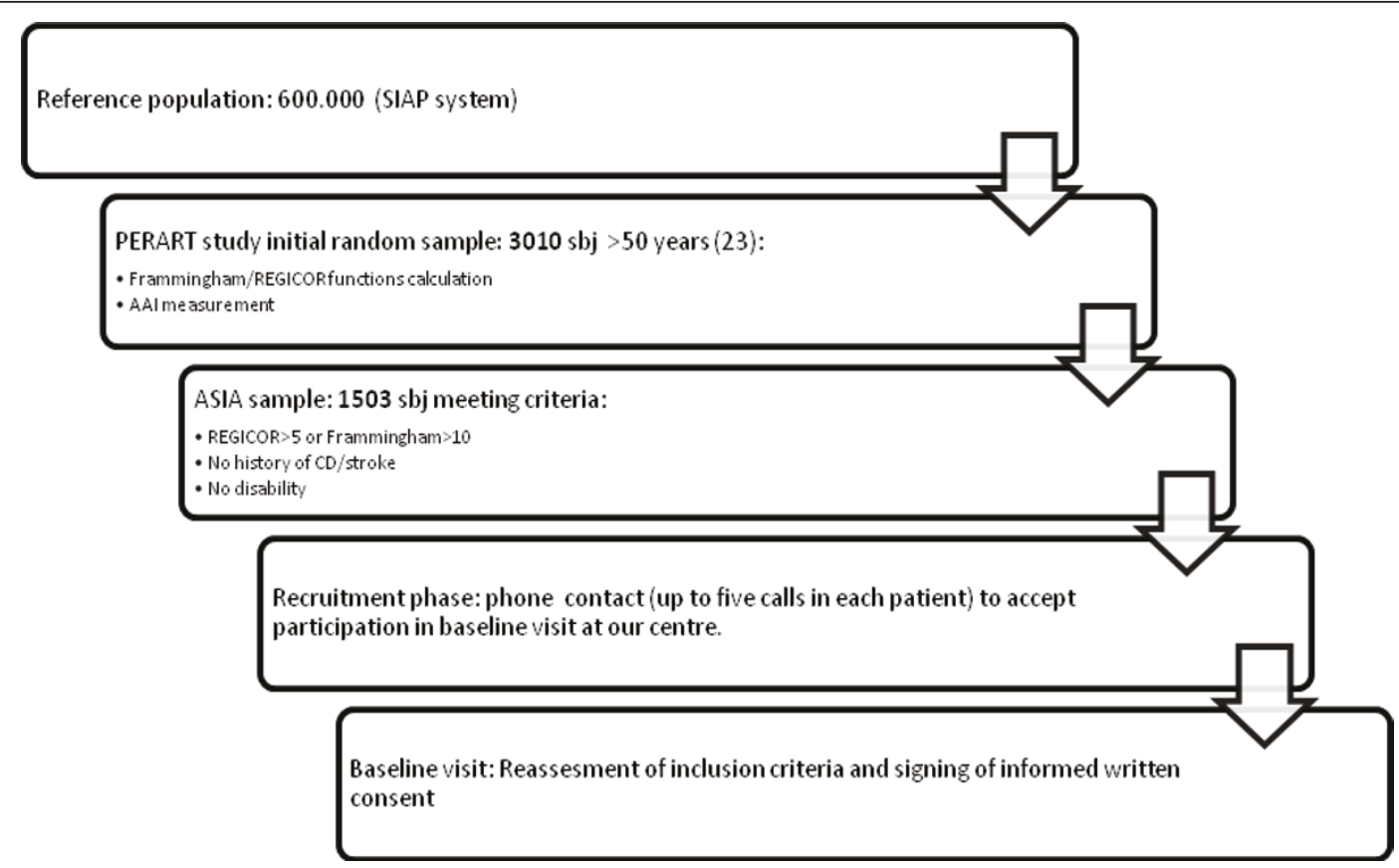

Figure 1 ASIA study sample selection. The diagram shows the sample selection in ASIA study from the reference population. SIAP system: Primary Care Information Technology System; sbj: subjects; AAl: ankle-arm index; CD: coronary disease.

- Sociodemographic variables: age, sex, socioeconomic status (monthly income in the family unit), education status (years of schooling, grade), employment (active, unemployed, retired, disqualified, housewife).

- Vascular risk factors: smoking habit (never, current, former), alcohol intake (g/day), physical activity level (sedentary, home-activity, outdoor activity and grade, walked $\mathrm{Km} /$ day in the last week), personal and family history of hypertension, diabetes mellitus and dyslipidemia.

- Current drug intake (platelet inhibitors, anticoagulants, lipid-lowering drugs, antihypertensives, hypoglycemic agents, antideppresants, etc).

- Vascular events presented between inclusion in the PERART study and baseline visit at our centre will be recorded: angina, myocardial infarction, intermittent claudication. Incidental stroke before the ASIA baseline visit was an exclusion criteria.

- Anthropometric variables: height, weight, waist circumference, current systolic/diastolic blood pressure values in both arms

- Ankle-arm index (AAI) was measured in all participants in PERART study as previously described (25).

- Others: Personal history of anxiety or depression, pharmacologic treatments in the moment of inclusion.
3. Biological and genetic studies. Twelve serum, 2 citrated plasma, 2 plasma-EDTA and 2 total blood cryotubes will be collected, processed and stored in each subject at baseline visit after a minimum of 12 fasting hours. After centrifugation at $3500 \mathrm{rpm}$ and $4^{\circ} \mathrm{C}$ for 15 minutes, serum or plasma will be blind coded and stored at $-80^{\circ} \mathrm{C}$ until analyzed.

4. Ultrasound protocol. All the Duplex studies will be performed in the same ultrasound lab (Neuroscience Department of Germans Trias i Pujol Hospital) using a General Electrid Vivid/Pro (GE Vingamed Ultrasound, Horten, Norway), by two experienced neurologists. First, a cervical study will be performed to detect presence and severity of atherosclerotic plaques in carotid arteries and origin of vertebral arteries, and to measure the intimamedia thickness (IMT). Later, a transcranial duplex will be set to examine bilateral intracranial carotid artery (ICA), middle cerebral artery (MCA), anterior cerebral artery (ACA), posterior cerebral artery (PCA), vertebral artery (VA) and basilar artery (BA). All studies will be performed with subject in supine position.

a. Extracraneal study: It will be performed using a linear transducer $5-15 \mathrm{~Hz}$ (predefined parameters: $8.5 \mathrm{MHz}, 4 \mathrm{~cm}$ focus, FPS 63.8).

- Carotid assessment: First, a high resolution B-mode study will be conducted in longitudinal 


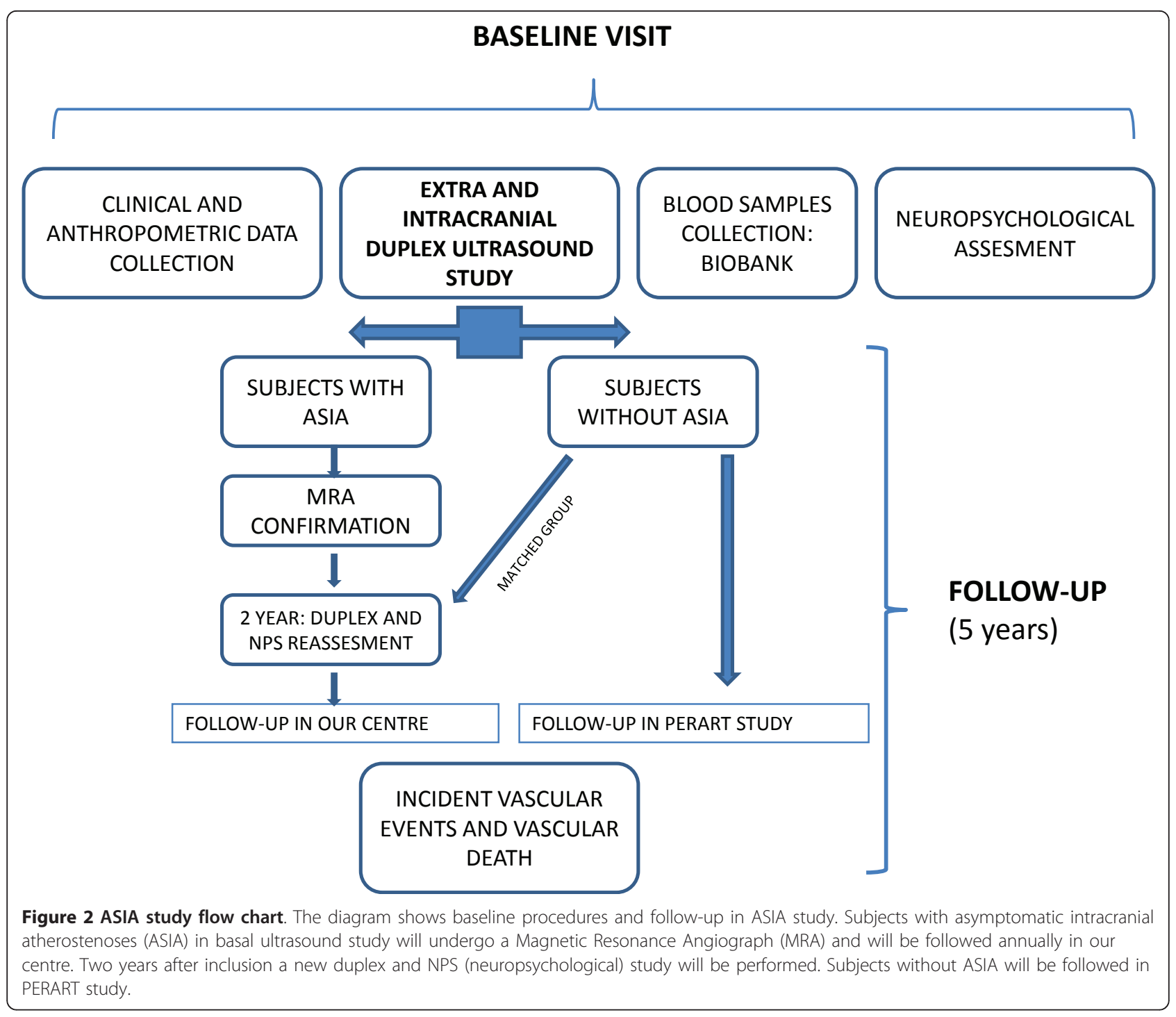

and cross-sectional planes over the whole visible length of the following arterial segments: proximal and distal common carotid artery, carotid bifurcation and internal carotid artery. The most pathologic areas in every arterial segment will be analyzed and images stored.

- Atherosclerotic plaques. According to Manheim consensus criteria, atherosclerotic plaques are defined as follows: focal structure encroaching into the arterial lumen of at least $0.5 \mathrm{~mm}$ or $50 \%$ of the surrounding IMT value, or demonstrating a thickness $>1.5 \mathrm{~mm}$ as measured from the media-adventitia interface to the intima-lumen interface [23]. Atherosclerotic plaques will be further characterized by the following criteria:

- Plaque surface: regular smooth, irregular, ulcerated [24]
- Echogenicity: anechogenic, hypo-anechogenic, hypo-isoechogenic, iso-hyperechogenic.

- IMT. According to Manheim consensus criteria, IMT is defined as a double-line pattern (the interface between lumen-intima and interface between media-adventitia) visualized by $\mathrm{B}$-mode sonography on both walls of the carotid arteries in a longitudinal image. IMT will be measured in regions without atherosclerotic plaque, preferably in CCA, in the far wall and more than $10 \mathrm{~mm}$ away from carotid bifurcation. Two values will be obtained: manual measurement of maximum IMT and automated measurement of mean IMT.

Next, Doppler spectrum analyses will be performed to assess the grade of stenosis depending on the systolic peak velocity [25]: $<50 \%:<125$ $\mathrm{cm} / \mathrm{s} ; 50-70 \%: 125-230 \mathrm{~cm} / \mathrm{s} ;>70 \%$ : $>230 \mathrm{~cm} / \mathrm{s}$. 
Significant carotid atherosclerosis will be considered when the stenosis is $\geq 50 \%$. In stenosis < $50 \%$ the percentage of luminal obstruction in axial plane will be calculated.

- Vertebral arteries: Extracranial vertebral arteries will be studied in the following segments: V2 segment (longitudinal insonation plane, framed by acoustical shadow of transverse processes); V0/V1 segment (origin from subclavian artery); and V3 segment (transverse insonation below mastoid bone, comma-shaped). We will characterize vertebral arteries following spectrum analyses [26] as normal, stenosis $>50 \%$ (SP > 125 $\mathrm{cm} / \mathrm{s}$ ) or occlusion (absence of telediastolic flow). b. Transcranial color-coded-duplex (TCCD) study: It will be performed using a 1.6-3.2 $\mathrm{MHz}$ transducer via transtemporal and transforaminal windows (patient in supine position) to evaluate circle of Willis and its branches, following consensus recommendations for an optimal exploration [27]. Each large cerebral artery will be investigated by spectral Doppler sonography with the color-coded Doppler signal used as a "road map". Flow direction, peak systolic (PSV), mean flow (MFV) and end-diastolic (PDV) velocities, pulsatility index (PI) and resistance index (RI) will be noted for every insonated artery. Angle correction will be performed when the Doppler sample volume is located within a straight vessel segment of at least $15-20 \mathrm{~mm}$. By transtemporal bone window, we will study intracranial carotid artery (ICA) in axial and coronal planes, middle cerebral artery (M1 and M2 segments), anterior cerebral artery (A1 segment) and posterior cerebral artery (P1 and P2 segments). By transforaminal bone window we will study vertebral artery (V4 segment) and basilar artery (BA). If there is an insufficient acoustic window in transcranial examination, an enhance contrast agent will be used intravenously (Sonovue ${ }^{\circledR}$ )

\section{Intracranial stenosis definition and grading}

An intracranial stenosis will be diagnosed following previous published criteria. First, using color-coded mode as a road map we will assess flow direction and presence of segmental color aliasing phenomena in all arteries. Then, we will determine the presence of an intracranial stenosis if the spectral analysis shows a focal increase of PSV and/or PDV higher than the mean value +2 SD for the corresponding cerebral artery or low-frequency, high-intensity Doppler signals, spectral widening or musical murmurs [28]. An example of intracranial stenosis is illustrated in figure 3. ICA stenosis will be diagnosed following published reference velocity values and recommendations, assessing not only differences in velocities between the two sides but also looking for activation of collaterals (anterior and/or posterior communicating arteries) and/or flow repercussion in extracranial internal carotid [29-31]. Following systolic peak criteria MCA stenosis will be graded into lowgrade $(140-209 \mathrm{~cm} / \mathrm{s})$, moderate $(210-279 \mathrm{~cm} / \mathrm{s})$ and high-grade $(>280 \mathrm{~cm} / \mathrm{s})$ [32]. For the rest of intracranial arteries we will follow cut-off values of PSV for $<50 \%$ and $\geq 50 \%$ stenosis [33]: $\geq 120 / \geq 155 \mathrm{~cm} / \mathrm{s}$ for anterior cerebral artery; $\geq 100 / \geq 145 \mathrm{~cm} / \mathrm{s}$ for posterior cerebral

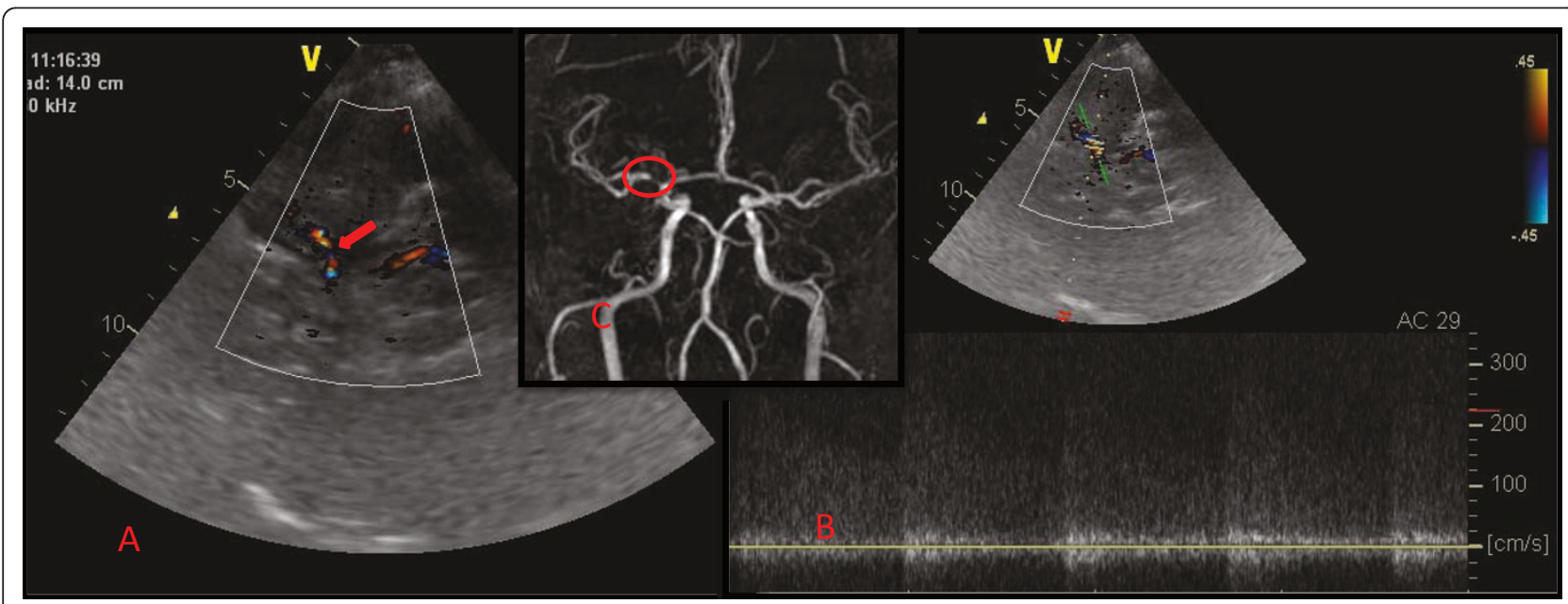

Figure 3 Example of right middle cerebral artery (MCA) stenosis. In left image (A) TCCD color mode shows an area of narrowing and color aliasing in the proximal segment of right MCA (arrow). In the right image (B), an spectral flow analyses is set to determine systolic peak velocity and appearance of low-frequency, high-intensity Doppler signals. In the middle superimposed image (C), a magnetic resonance angiogram (MRA) made in the same subject shows a flow gap in right MCA (circle) corresponding to a high-grade stenosis. 
and basilar arteries and $\geq 90 / \geq 120 \mathrm{~cm} / \mathrm{s}$ for vertebral artery. Furthermore, when a focal increase of velocity is detected, the proximal and distal vessel segments will be evaluated (pre-stenotic and post-stenotic flow patterns in the upstream and downstream vessel segments) and potential collateral pathways will be considered in order to assess hemodynamic repercussion. Number, location and severity of intracranial stenosis will be recorded in every subject.

\section{Neuropsychological assessment}

Neuropsychological studies will be performed at baseline visit in our centre after accepting and signing specific informed written consent. Neuropsychological studies will be performed by neuropsychologists blinded to neurosonology study results. General cognitive status will be measured using the Mini Mental State Examination. Depressive symptoms will be assessed with the Geriatric Depression Scale with scores higher than 5 being indicative of probable depression. Cognitive measures will assess executive functions, attention, verbal and visual memory, visuoconstructive abilities, speed/visuomotor coordination and language.

\section{Magnetic Resonance (MR) and MR-angiography (MRA) study}

A complete MR will be conducted to patients with stenosis detected by TCCD if they have no contraindication. All explorations will be performed with the same 1.5T MR (Intera, Philips), with an echo-planar capacity of $25 \mathrm{~m}$ Teslas/m and time-rises of 300-350 microseconds. The MRA will be performed by a time-of-flight (TOF) sequence, using $1.5 \mathrm{~mm}$ section slides, 200-mm field of view, $200 \times$ 512 matrix, and 7-11 minutes of acquisition. Maximal intensity projection (MIP) reconstructions will be set.

All MR studies will be performed in a 6 months maximum period from baseline inclusion visit (sonographic study). MRAs will be analyzed by a neuroradiologist blind to clinical and sonographic data. Intracranial stenosis will be defined as flow irregularity and focal narrowing $>50 \%$ in luminal reduction affecting the main cerebral arteries.

\section{Primary prevention strategies}

- Vascular risk factors control (obesity, hypertension, dyslipidemia, hyperglycemia, sedentarism, smoking habit...). The family doctor will assume their control, after receiving a brief report of the neurosonolgical study and the clinical data obtained in the baseline visit in our centre.

- Antiplatelet treatment: After neurosonological study, antithrombotic treatment (300 mg of aspirin or $75 \mathrm{mg}$ of clopidogrel-if there is intolerance/allergy to aspirin-) will be recommended according to
AHA/ASA primary prevention guidelines [34] in the following cases: asymptomatic carotid stenosis $>50 \%$; significant stenosis of extracranial vertebral arteries and/or asymptomatic intracranial stenosis.

- Carotid revascularization: Following the Asymptomatic Carotid Atherosclerosis Study (ACAS) [35], carotid revascularization (endarterectomy or endovascular) will be recommended in carotid stenosis $>60 \%$, performed by surgeons or interventional neuroradiologists with periprocedural complication rates lower than $3 \%$.

\section{ASIA clinical and image database}

Clinical data will be recorded in CRFs. Blood samples will be processed, frozen and stored in our biobank. Images obtained in neurosonological studies will be stored in a specific workstation (Echo Pack) to be analyzed afterwards. A prospective electronic database will be created with clinical, neurosonological and laboratory variables of the cross-over and longitudinal phases of the study.

\section{Prospective follow-up (cohort study)}

Participants will be followed-up annually for 5 years by primary care physicians of the PERART study to document the incidence of vascular events, therapeutic compliance and control of vascular risk factors. The primary endpoint will be the combined incidence of any major vascular event: acute myocardial infarction or angina requiring hospitalization, ischemic stroke, hemorrhagic stroke and vascular death.

Subjects with intracranial stenosis at baseline will be controlled in the Germans Trias i Pujol University Hospital annually. A new neurosonological and neuropsychological study will be performed two years after inclusion to study progression or regression of the intracranial lesions and cognitive decline. In addition, a matched group of subjects without baseline ASIA will be studied. Vascular events will be adjudicated by an external monitoring committee comprising of two neurologists and one cardiologist.

\section{Satatistical considerations}

Statistics will be performed with the SPSS 18.0 statistical package. Quantitative variables will be compared with the Student's t test and analysis of variance will be performed, using the corresponding non parametric tests when necessary. Chi squared test will be used for comparisions of categorical variables. In the cross-over phase, multiple logistic regression models will be performed to identify variables independently associated with the presence of asymptomatic intracranial atherostenoses. In longitudinal phase, survival analysis for the 
combination of major vascular events and for each vascular event will be performed with the Kaplan-Meier curves according to the presence/absence of intracranial stenosis. Cox multivariate regression models will be used to compare the probability of having a vascular event in the follow up cohorts, adjusting for the necessary covariates. The relative risk (hazards ratios) will be given whit their corresponding 95\% confidence intervals.

\section{Discussion}

Despite of its relevant impact on public health and the development of new non-invasive diagnostic tests, intracranial atherosclerosis is still an understudied pathology. ICAD is the origin of $5-10 \%$ of strokes in Caucasians $[36,37]$ and up to $50 \%$ in Asians [38,39]. Limited data about preclinical stage and natural history of ICAD are available in the general population. The only population studies to determine its prevalence in stroke-free individuals were developed in Asians without prospective follow-up to assess the risk of vascular events (21-25). These studies have some limitations as they evaluated intracranial stenosis only with transcranial Doppler, they did not use contrast agents and some of them only evaluated middle cerebral artery. There is too much variability in their methods to compare them and to establish a real prevalence. Our study is the first to evaluate the prevalence of asymptomatic intracranial atherosclerosis in Caucasians. As it is a cohort study we will also investigate the ASIA prognosis impact, not only in the appearance of cerebral events but also in coronary and peripheral artery disease, and in the development of vascular cognitive impairment.

We will determine clinical, instrumental (IMT and AAI measurements) and biological risk factors related to asymptomatic ICAD in order to identify factors associated with its progression and clinical expression. We will be able to analyze whether factors that have been already related to disease progression in its symptomatic stage, such as inflammatory markers, also pay a role in asymptomatic intracranial atherosclerosis [40-43]. Furthermore, we would evaluate the importance of using those risk factors as risk markers in terms of general vascular primary prevention.

The reasons that explain the racial and interindividual differences observed in the distribution of cerebral atherosclerosis affecting extra and intracranial arteries remain poorly understood. Differences in vascular risk factors profile, inflammatory markers, life-style and genetic susceptibility are proposed as possible answers but studies are contradictory. Our study could contribute to clarify this matter since we will evaluate extracranial and intracranial vasculature in each patient.

Finally, many studies are stressing the possible relationship between ICAD and cognitive impairment.
A simultaneous protocol in collaboration with our neuropsychological team has been created to evaluate cognitive status in every subject of ASIA study. Consequently, the ASIA results may help to find out biological, sonographic and clinical factors related to the presence of vascular cognitive impairment in asymptomatic individuals.

Strengths of this study are: 1). Randomized, large sample population study that will provide a generalized estimation of prevalence of asymptomatic intracranial stenosis in Caucasians, which nowadays remains unknown; 2). TCCD assessment of all intracranial arteries (anterior and posterior circulation) in all subjects, use of contrast agents if insufficient acoustic window and MRA assessment in subjects with stenosis; 3). Complete cerebral neurosonology study in each patient (intracranial and extracranial) because an adequate interpretation of intracranial findings always requires a careful assessment of the influence of extracranial pathology on the intracranial hemodynamics; 4). A biobank will be created allowing investigation of molecular and genetic factors related to the presence and progression of intracranial atherosclerosis; 5). Long-term follow-up that will allow us to establish prognosis and related factors of incidental stroke and major vascular events, and to evaluate progression/regression of intracranial lesions.

We hope ASIA study will contribute to the better understanding of intracranial atherosclerotic disease dynamics and help us to identify new potential therapeutic and prevention targets for this condition.

\section{Funding support}

This project is supported by the program of Promotion in the Biomedical Investigation and Health Sciences from the Carlos III Health Institute of the Spanish Health and Social Policy Ministry [PI070393]. Dr. López-Cancio is a neurologist granted with a Río Hortega research contract from the Carlos III Health Institute of the Spanish Health and Social Policy Ministry, co-financed by the Germans Trias i Pujol Research Institute Foundation. Neuropsychological studies are supported by the Juan de la Cierva research program of the Spanish Health and Social Policy Ministry [SEJ2006-15399/PSIC].

\section{Acknowledgements}

We gratefully thank investigators of PERART study as they provided us the randomized sample of subjects meeting criteria for our study, the data needed to calculate vascular risk functions and the AAI measurements. We also thank administratives of our Neurology department for undertaking the telephone calls and programming the clinic visits.

\section{Author details}

'Department of Neurosciences, Hospital Universitari Germans Trias I Pujol, Universitat Autònoma de Barcelona, Badalona, Barcelona, Spain. ${ }^{2}$ Magnetic Resonance Unit, Hospital Universitari Germans Trias I Pujol, Universitat 
Autònoma de Barcelona, Badalona, Barcelona, Spain. ${ }^{3}$ Department of Psychiatry and Clinical Psychobiology, Universitat de Barcelona, Barcelona (Spain. ${ }^{4}$ Department of Biochemistry, Hospital Universitari Germans Trias I Pujol, Universitat Autònoma de Barcelona, Badalona, Barcelona, Spain. ${ }^{5}$ Primary Healthcare Research Support Unit Metropolitana Nord, ICS-IDIAP Jordi Gol, Mataró, Barcelona, Spain. ${ }^{6}$ Neurology Service, Stroke Unit, Hospital Clínico Universitario, Valladolid, Spain.

\section{Authors' contributions}

JFA conceived of and designed the study and is the principal investigator; AD is the Neurosciences Department of Germans Trias I Pujol Hospital director, participated in study design, have made a major revision of this manuscript and will provide all logistic support; ELC will carry out subject basal visits, ultrasonographic studies, database maintenance, subjects followup, coordination with other departments and wrote the initial draft of this article; LD will carry out patient basal visits and ultrasonographic studies; AS and SR will carry out the anthropometric and blood pressure measurements in basal visit and the processing and storage of biobank; AG will carry out molecular and bioquemistry studies. MM will coordinate all neuropsychological studies; MA, PT and GP provided randomized sample of subjects and clinical data from PERART study and will coordinate the followup study in primary care network. All authors read and approved the final manuscript.

\section{Competing interests}

The authors declare that they have no competing interests.

Received: 8 November 2010 Accepted: 17 February 2011 Published: 17 February 2011

\section{References}

1. Gorelick PB, Wong KS, Bae HJ, Pandey DK: Large artery intracranial occlusive disease: A large worldwide burden but a relatively neglected frontier. Stroke 2008, 39(8):2396-9.

2. Roher AE, Garami Z, Alexandrov AV, Kokjohn TA, Esh CL, Kalback WM, Vedders $L$, Wilson JR, Sabbagh MN, Beach TG: Interaction of cardiovascular disease and neurodegeneration: Transcranial doppler ultrasonography and alzheimer's disease. Neurol Res 2006, 28(6):672-8.

3. Beach TG, Wilson JR, Sue LI, Newell A, Poston M, Cisneros R, Pandya $Y$, Esh C, Connor DJ, Sabbagh M, Walker DG, Roher AE: Circle of willis atherosclerosis: Association with alzheimer's disease, neuritic plaques and neurofibrillary tangles. Acta Neuropathol 2007, 113(1):13-21.

4. Kalaria RN, Maestre GE, Arizaga R, Friedland RP, Galasko D, Hall K, Luchsinger JA, Ogunniyi A, Perry EK, Potocnik F, Prince M, Stewart R, Wimo A, Zhang ZX, Antuono P, World Federation of Neurology Dementia Research Group: Alzheimer's disease and vascular dementia in developing countries: Prevalence, management, and risk factors. Lancet Neurol 2008, 7(9):812-26.

5. ladecola C: The overlap between neurodegenerative and vascular factors in the pathogenesis of dementia. Acta Neuropathol 2010.

6. Mazighi M, Tanasescu R, Ducroca X, Vicaut E, Bracard S, Houdart E, Woimant F: Prospective study of symptomatic atherothrombotic intracranial stenoses: The GESICA study. Neurology 2006, 66(8):1187-91.

7. Chimowitz MI, Lynn MJ, Howlett-Smith H, Stern BJ, Hertzberg VS, Frankel MR, Levine SR, Chaturvedi S, Kasner SE, Benesch CG, Sila CA, Jovin TG, Romano JG, Warfarin-Aspirin Symptomatic Intracranial Disease Trial Investigators: Comparison of warfarin and aspirin for symptomatic intracranial arterial stenosis. N Engl J Med 2005, 352(13):1305-16.

8. Kern R, Steinke W, Daffertshofer M, Prager R, Hennerici M: Stroke recurrences in patients with symptomatic vs asymptomatic middle cerebral artery disease. Neurology 2005, 859-64.

9. Kasner SE, Chimowitz MI, Lynn MJ, Howlett-Smith H, Stern BJ, Hertzberg VS, Frankel MR, Levine SR, Chaturvedi S, Benesch CG, Sila CA, Jovin TG, Romano JG, Cloft HJ, Warfarin Aspirin Symptomatic Intracranial Disease Trial Investigators: Predictors of ischemic stroke in the territory of a symptomatic intracranial arterial stenosis. Circulation 2006, 113(4):555-63.

10. Kasner SE, Lynn MJ, Chimowitz MI, Frankel MR, Howlett-Smith H, Hertzberg VS, Chaturvedi S, Levine SR, Stern BJ, Benesch CG, Jovin TG, Sila CA, Romano JG, Warfarin Aspirin Symptomatic Intracranial Disease (WASID) Trial Investigators: Warfarin vs aspirin for symptomatic intracranial stenosis: Subgroup analyses from WASID. Neurology 2006, 67(7):1275-8.

11. Derdeyn CP, Chimowitz Ml: Angioplasty and stenting for atherosclerotic intracranial stenosis: Rationale for a randomized clinical trial. Neuroimaging Clin N Am 2007, 17(3):355, 63, viii-ix.

12. Arenillas JF, Candell-Riera J, Romero-Farina G, Molina CA, Chacon P, Aguade-Bruix S, Montaner J, de Leon G, Castell-Conesa J, Alvarez-Sabin J: Silent myocardial ischemia in patients with symptomatic intracranial atherosclerosis: Associated factors. Stroke 2005, 36(6):1201-6.

13. Hoshino A, Nakamura T, Enomoto S, Kawahito H, Kurata H, Nakahara Y, ljichi T: Prevalence of coronary artery disease in japanese patients with cerebral infarction: Impact of metabolic syndrome and intracranial large artery atherosclerosis. Circ J 2008, 72(3):404-8.

14. Naghavi M, Libby P, Falk E, Casscells SW, Litovsky S, Rumberger J, Badimon JJ, Stefanadis C, Moreno P, Pasterkamp G, Fayad Z, Stone PH, Waxman S, Raggi P, Madjid M, Zarrabi A, Burke A, Yuan C, Fitzgerald PJ, Siscovick DS, de Korte CL, Aikawa M, Juhani Airaksinen KE, Assmann G, Becker CR, Chesebro JH, Farb A, Galis ZS, Jackson C, Jang IK, et al: From vulnerable plaque to vulnerable patient: A call for new definitions and risk assessment strategies: Part I. Circulation 2003, 108(14):1664-72.

15. Naghavi M, Libby P, Falk E, Casscells SW, Litovsky S, Rumberger J, Badimon JJ, Stefanadis C, Moreno P, Pasterkamp G, Fayad Z, Stone PH, Waxman S, Raggi P, Madjid M, Zarrabi A, Burke A, Yuan C, Fitzgerald PJ, Siscovick DS, de Korte CL, Aikawa M, Airaksinen KE, Assmann G, Becker CR, Chesebro JH, Farb A, Galis ZS, Jackson C, Jang IK, et al: From vulnerable plaque to vulnerable patient: A call for new definitions and risk assessment strategies: Part II. Circulation 2003, 108(15):1772-8.

16. Marrugat J, Sala J: New instruments, same risks. Rev Esp Cardiol 2007, 60(5):464-7.

17. Huang HW, Guo MH, Lin RJ, Chen YL, Luo Q, Zhang Y, Wong KS: Prevalence and risk factors of middle cerebral artery stenosis in asymptomatic residents in rongqi county, guangdong. Cerebrovasc Dis 2007, 24(1):111-5.

18. Wong KS, Huang YN, Yang HB, Gao S, Li H, Liu JY, Liu Y, Tang A: A door-todoor survey of intracranial atherosclerosis in liangbei county, china. Neurology 2007, 68(23):2031-4.

19. Wong KS, Ng PW, Tang A, Liu R, Yeung V, Tomlinson B: Prevalence of asymptomatic intracranial atherosclerosis in high-risk patients. Neurology 2007, 68(23):2035-8.

20. Bae HJ, Lee J, Park JM, Kwon O, Koo JS, Kim BK, Pandey DK: Risk factors of intracranial cerebral atherosclerosis among asymptomatics. Cerebrovasc Dis 2007, 24(4):355-60.

21. Alzamora MT, Baena-Diez JM, Sorribes M, Fores $R$, Toran P, Vicheto M, Pera G, Reina MD, Albaladejo C, Llussa J, Bundo M, Sancho A, Heras A Rubies J, Arenillas JF, PERART study: Peripheral arterial disease study (PERART): Prevalence and predictive values of asymptomatic peripheral arterial occlusive disease related to cardiovascular morbidity and mortality. BMC Public Health 2007, 7:348.

22. Marrugat J, Subirana I, Comin E, Cabezas C, Vila J, Elosua R, Nam BH, Ramos R, Sala J, Solanas P, Cordon F, Gene-Badia J, D'Agostino RB, VERIFICA Investigators: Validity of an adaptation of the framingham cardiovascular risk function: The VERIFICA study. J Epidemiol Community Health 2007. 61(1):40-7.

23. Touboul PJ, Hennerici MG, Meairs $S$, Adams $H$, Amarenco $P$, Bornstein $N$, Csiba L, Desvarieux M, Ebrahim S, Fatar M, Hernandez Hernandez R, Jaff M, Kownator S, Prati P, Rundek T, Sitzer M, Schminke U, Tardif JC, Taylor A, Vicaut E, Woo KS, Zannad F, Zureik M: Mannheim carotid intima-media thickness consensus (2004-2006). an update on behalf of the advisory board of the 3rd and 4th watching the risk symposium, 13th and 15th european stroke conferences, mannheim, germany, 2004, and brussels, belgium, 2006. Cerebrovasc Dis 2007, 23(1):75-80.

24. Prabhakaran S, Rundek T, Ramas R, Elkind MS, Paik MC, Boden-Albala B, Sacco RL: Carotid plaque surface irregularity predicts ischemic stroke: The northern manhattan study. Stroke 2006, 37(11):2696-701.

25. Grant EG, Benson CB, Moneta GL, Alexandrov AV, Baker JD, Bluth El, Carroll BA, Eliasziw M, Gocke J, Hertzberg BS, Katanick S, Needleman L, Pellerito J, Polak JF, Rholl KS, Wooster DL, Zierler RE: Carotid artery stenosis: Gray-scale and doppler US diagnosis-society of radiologists in ultrasound consensus conference. Radiology 2003, 229(2):340-6. 
26. de Bray JM, Pasco A, Tranquart F, Papon X, Alecu C, Giraudeau B, Dubas F, Emile $\mathrm{J}$ : Accuracy of color-doppler in the quantification of proximal vertebral artery stenoses. Cerebrovasc Dis 2001, 11(4):335-40.

27. Nedelmann M, Stolz E, Gerriets T, Baumgartner RW, Malferrari G, Seidel G, Kaps M, TCCS Consensus Group: Consensus recommendations for transcranial color-coded duplex sonography for the assessment of intracranial arteries in clinical trials on acute stroke. Stroke 2009, 40(10):3238-44.

28. Lin SK, Ryu SJ, Chang YJ, Lee TH: Clinical relevance of musical murmurs in color-coded carotid and transcranial duplex sonographies. AJNR Am J Neuroradiol 2006, 27(7):1493-7.

29. Valaikiene J, Schuierer G, Ziemus B, Dietrich J, Bogdahn U, Schlachetzki F: Transcranial color-coded duplex sonography for detection of distal internal carotid artery stenosis. AJNR Am J Neuroradiol 2008, 29(2):347-53.

30. Valdueza JM, Schreiber SJ, Rohel JE, Klingebiel R: Vascular pathology. In Neurosonology and Neuroimaging of Stroke. 2008th. Edited by: Thieme Verlag G. Sttutgart: Thieme; 2008:76-96.

31. Ley-Pozo J, Ringelstein EB: Noninvasive detection of occlusive disease of the carotid siphon and middle cerebral artery. Ann Neurol 1990, 28(5):640-7.

32. Röther J, Schwartz AS, Wentz K, Hennerici M: Middle cerebral artery stenoses: Assessment by magnetic resonance angiography and transcranial doppler ultrasound. Cerebrovasc Dis 1994, 4:273-9.

33. Baumgartner RW, Mattle HP, Schroth G: Assessment of $>/=50 \%$ and $<50 \%$ intracranial stenoses by transcranial color-coded duplex sonography. Stroke 1999, 30(1):87-92.

34. Goldstein LB, Adams R, Alberts MJ, Appel LJ, Brass LM, Bushnell CD, Culebras A, Degraba TJ, Gorelick PB, Guyton JR, Hart RG, Howard G, KellyHayes M, Nixon JV, Sacco RL, American Heart Association/American Stroke Association Stroke Council, Atherosclerotic Peripheral Vascular Disease Interdisciplinary Working Group, Cardiovascular Nursing Council, Clinical Cardiology Council, Nutrition, Physical Activity, and Metabolism Council, Quality of Care and Outcomes Research Interdisciplinary Working Group, American Academy of Neurology. Primary prevention of ischemic stroke: A guideline from the american heart Association/American stroke association stroke council: Cosponsored by the atherosclerotic peripheral vascular disease interdisciplinary working group; cardiovascular nursing council; clinical cardiology council; nutrition, physical activity, and metabolism council; and the quality of care and outcomes research interdisciplinary working group: The american academy of neurology affirms the value of this guideline. Stroke 2006, 37(6):1583-633.

35. Endarterectomy for asymptomatic carotid artery stenosis: executive committee for the asymptomatic carotid atherosclerosis study. JAMA 1995, 273(18):1421-8.

36. Wityk RJ, Lehman D, Klag M, Coresh J, Ahn H, Litt B: Race and sex differences in the distribution of cerebral atherosclerosis. Stroke 1996, 27(11):1974-80.

37. Sacco RL, Kargman DE, Gu Q, Zamanillo MC: Race-ethnicity and determinants of intracranial atherosclerotic cerebral infarction. the northern manhattan stroke study. Stroke 1995, 26(1):14-20.

38. Wong KS, Huang YN, Gao S, Lam WW, Chan YL, Kay R: Intracranial stenosis in chinese patients with acute stroke. Neurology 1998, 50(3):812-3.

39. Huang YN, Gao S, Li SW, Huang Y, Li JF, Wong KS, Kay R: Vascular lesions in chinese patients with transient ischemic attacks. Neurology 1997, 48(2):524-5.

40. Arenillas JF, Alvarez-Sabin J, Molina CA, Chacon P, Montaner J, Rovira A, Ibarra B, Quintana M: C-reactive protein predicts further ischemic events in first-ever transient ischemic attack or stroke patients with intracranial large-artery occlusive disease. Stroke 2003, 34(10):2463-8.

41. Arenillas JF, Alvarez-Sabin J: Basic mechanisms in intracranial large-artery atherosclerosis: Advances and challenges. Cerebrovasc Dis 2005, 20(Suppl 2):75-83.

42. Arenillas JF, Alvarez-Sabin J, Molina CA, Chacon P, Fernandez-Cadenas I, Ribo M, Delgado P, Rubiera M, Penalba A, Rovira A, Montaner J: Progression of symptomatic intracranial large artery atherosclerosis is associated with a proinflammatory state and impaired fibrinolysis. Stroke 2008, 39(5):1456-63.

43. Arenillas JF, Molina CA, Chacon P, Rovira A, Montaner J, Coscojuela P, Sanchez E, Quintana M, Alvarez-Sabin J: High lipoprotein (a), diabetes, and the extent of symptomatic intracranial atherosclerosis. Neurology 2004, 63(1):27-32.

\section{Pre-publication history}

The pre-publication history for this paper can be accessed here: http://www.biomedcentral.com/1471-2377/11/22/prepub

doi:10.1186/1471-2377-11-22

Cite this article as: López-Cancio et al:: The population-based BarcelonaAsymptomatic Intracranial Atherosclerosis Study (ASIA): rationale and design. BMC Neurology 2011 11:22.

\section{Submit your next manuscript to BioMed Central and take full advantage of:}

- Convenient online submission

- Thorough peer review

- No space constraints or color figure charges

- Immediate publication on acceptance

- Inclusion in PubMed, CAS, Scopus and Google Scholar

- Research which is freely available for redistribution 